\title{
The recurrence dimension for piecewise monotonic maps of the interval
}

\author{
FRANZ HOFBAUER
}

\begin{abstract}
We investigate a weighted version of Hausdorff dimension introduced by V. Afraimovich, where the weights are determined by recurrence times. We do this for an ergodic invariant measure with positive entropy of a piecewise monotonic transformation on the interval $[0,1]$, giving first a local result and proving then a formula for the dimension of the measure in terms of entropy and characteristic exponent. This is later used to give a relation between the dimension of a closed invariant subset and a pressure function.
\end{abstract}

Mathematics Subject Classification (2000): 37E05 (primary); 37C45, 28A80, 37B40, 28A78 (secondary).

\section{Introduction}

In the last years there was much interest in generalized notions of dimension. In [14] a theory of dimension structures is developed. Special cases of these structures are weighted versions of Hausdorff dimensions. We define them only on the interval $[0,1]$. Let $|U|$ be the diameter of a subset $U$ of $[0,1]$. For $\delta>0$ a $\delta$-cover of a set $A \subset[0,1]$ is a finite or countable collection of intervals of length $\leq \delta$ covering $A$. For a set function $w$ with values in $\mathbb{R}^{+}$and $s, t \in \mathbb{R}$ we define for $A \subset[0,1]$

$$
v_{s, t}(A)=\lim _{\delta \rightarrow 0} \inf _{\mathcal{C}} \sum_{U \in \mathcal{C}} w(U)^{s}|U|^{t}
$$

where the infimum is taken over all $\delta$-covers $\mathcal{C}$ of $A$. It follows from the general theory in [14] that for fixed $s \in \mathbb{R}$ there is a critical value $t_{c} \in[-\infty, \infty]$ such that $v_{s, t}(A)=\infty$ for $t<t_{c}$ and $v_{s, t}(A)=0$ for $t>t_{c}$. We denote this critical value $t_{c}$ by $d_{s}(A)$ and call it the dimension of the set $A$.

If $m$ is a probability measure on $[0,1]$ and if one sets $w(U)=m(U)$, one gets the dimension introduced by Olsen in [13] and called multifractal generalization of

Research for this paper was partially carried out while the author was visiting the Max Planck Institut for Mathematics in Bonn as a participant in the activity on Algebraic and Topological Dynamics.

Pervenuto alla Redazione il 3 febbraio 2005 e in forma definitiva il 2 agosto 2005. 
the Hausdorff dimension there. For invariant sets of piecewise monotonic maps on the interval, this dimension is investigated in [9].

If $T:[0,1] \rightarrow[0,1]$ is a map, one can define the recurrence time of a set $U \subset[0,1]$ by $\tau(U)=\min \left\{n \geq 1: T^{n}(U) \cap U \neq \emptyset\right\}$. If one sets $w(U)=e^{-\tau(U)}$ one gets the dimension introduced in [1] (see also [2]) and called the spectrum of dimensions for Poincaré recurrences there. This is the dimension we consider in this paper. We call it recurrence dimension for simplicity.

For topological Markov chains and conformal repellers satisfying a specification property this recurrence dimension has been computed in terms of topological entropy or implicitly using a pressure function (see [2]). In this paper we do the same for invariant subsets of piecewise monotonic maps on the interval $[0,1]$. We say that $T:[0,1] \rightarrow[0,1]$ is piecewise monotonic if there are numbers $0=c_{0}<c_{1}<\cdots<c_{N}=1$, such that $T \mid\left(c_{i-1}, c_{i}\right)$ is monotone and continuous for $1 \leq i \leq N$. Set $\mathcal{Z}=\left\{\left(c_{0}, c_{1}\right), \ldots,\left(c_{N-1}, c_{N}\right)\right\}$ and $P=\left\{c_{0}, c_{1}, \ldots, c_{N}\right\}$. We assume that the dynamical system $([0,1], T)$ has only finitely many or no attracting periodic orbits. In chapter IV of [12] it shown that this holds for $C^{2}$-maps $T$ with non-flat critical points. In the second part of this paper we assume that $T$ is expanding, which implies that there can be no attracting periodic orbits. Furthermore, we assume that $T$ has a continuous derivative $T^{\prime}$ on $[0,1] \backslash P$ and set $\varphi=-\log \left|T^{\prime}\right|$. We shall need regularity conditions for $\varphi$. To this end let $C$ be the set of all functions $f:[0,1] \backslash P \rightarrow \mathbb{R}$, such that for each $Z \in \mathcal{Z}$ the map $f \mid Z$ can be extended to a continuous real-valued function on the closure $\bar{Z}$ of $Z$. Furthermore let $D$ be the set of all functions $f:[0,1] \backslash P \rightarrow \mathbb{R}$, such that for each $Z \in \mathcal{Z}$ the map $f \mid Z$ can be extended to a continuous function $f_{Z}: \bar{Z} \rightarrow(-\infty, \infty]$. For $p \geq 1$ let $D^{p}$ be the set of all $f \in D$ such that $e^{-f}$ is of bounded $p$-variation. The $p$-variation of a function $g:[0,1] \rightarrow \mathbb{R}$ is defined by $\operatorname{var}^{p} g:=\sup \left\{\sum_{j=1}^{n}\left|g\left(x_{j}\right)-g\left(x_{j-1}\right)\right|^{p}\right\}$ where the sup is taken over $n \geq 1$ and $0 \leq x_{0}<x_{1}<\cdots<x_{n} \leq 1$. Notice that $\varphi \in C$ excludes the possibility that $T^{\prime}$ attains the value zero, whereas this is possible on $P$, if $\varphi \in D$.

In the first part of the paper we investigate the dimension of an invariant measure. For a probability measure $\mu$ we define

$$
d_{S}(\mu)=\inf \left\{d_{S}(A): \mu(A)=1\right\} .
$$

Theorem 2.4 below gives a local result. If $\varphi$ is either in $C$ or in $D^{p}$ for some $p \geq 1$ and if $\mu$ is an ergodic $T$-invariant measure with $h_{\mu}>0$, then $\chi_{\mu}:=$ $-\mu(\varphi)$ is greater than zero and $\lim _{r \rightarrow 0} \frac{\tau\left(B_{r}(x)\right)}{-\log r}=\frac{1}{\chi_{\mu}}$ holds for $\mu$-almost all $x$. In this paper we prove only $\limsup _{r \rightarrow 0} \frac{\tau\left(B_{r}(x)\right)}{-\log r} \leq \frac{1}{\chi_{\mu}}$ using methods similar to those used in [7] and [8] for Hausdorff dimension and local dimension of an ergodic invariant measure. The other part of this theorem, namely $\liminf _{r \rightarrow 0} \frac{\tau\left(B_{r}(x)\right)}{-\log r} \geq$ $\frac{1}{\chi_{\mu}}$, is already shown in [15]. Using Theorem 2.4 and results from [7] on local dimension we get then that $d_{s}(\mu)=\frac{h_{\mu}-s}{\chi_{\mu}}$ for every $s \in\left[0, h_{\mu}\right)$. This is stated as 
Theorem 2.6 below. Similar results are known for subshifts with positive entropy satisfying a specification property (see [3]).

In the second part of this paper we investigate the dimension of a closed subset $A$ of $[0,1]$, which is invariant under the piecewise monotonic map $T$. We assume that $T$ is expanding, which means that inf $\left|T^{\prime}\right|>1$, and that $\varphi \in C$. Furthermore, we set $\mathcal{Z}_{n}=\bigvee_{j=0}^{n-1} T^{-j} \mathcal{Z}$. For a function $f \in C$ we define

$$
p(T \mid A, f)=\limsup _{n \rightarrow \infty} \frac{1}{n} \log \sum_{Z \in \mathcal{Z}_{n}, Z \cap A \neq \emptyset} \sup _{x \in Z \cap A} e^{\sum_{j=0}^{n-1} f\left(T^{j} x\right)} .
$$

This is the definition of pressure for the isomorphic shift space one gets by coding with respect to the partition $\mathcal{Z}$. Since the transformation $T$ can have discontinuities on the set $P$, we use $p(T \mid A, f)$ as the notion of pressure. Set $\pi(t)=p(T \mid A, t \varphi)$ for $t \geq 0$. For $s \in\left[0, h_{\text {top }}(T \mid A)\right)$ we show then that there is a unique solution $z_{s}(A)>0$ of the equation $\pi(t)=s$, which equals $d_{s}(A)$. This is stated as Theorem 3.1 below.

\section{Dimension of an invariant measure}

In order to investigate the recurrence dimension of an ergodic invariant measure $\mu$ with $h_{\mu}>0$ we use Markov extensions introduced in [5] and [10] and used in [7] and in [8] for the investigation of Hausdorff dimensions. Let $\mathcal{Y}$ be a finite or countable collection of open pairwise disjoint intervals, such that $T \mid Y$ is monotone for all $Y \in \mathcal{Y}$ and such that $\mu\left(\bigcup_{Y \in \mathcal{Y}} Y\right)=1$. Set $R=\bigcap_{j=0}^{\infty} T^{-j}\left(\bigcup_{Y \in \mathcal{Y}} Y\right)$. Since $\mu$ is invariant, we have $\mu(R)=1$. For $n \geq 1$ let $Y_{n}(x)$ be the unique element of $\mathcal{Y}_{n}=\bigvee_{j=0}^{n-1} T^{-j} \mathcal{Y}$, which contains $x$. If $x \in R$, then $Y_{n}(x)$ exists for all $n \geq 1$.

The Markov extension of the dynamical system $([0,1], \mu, T)$ with respect to $\mathcal{Y}$ is constructed as follows. If $D$ is a subinterval of some $Y \in \mathcal{Y}$, we call the nonempty sets among $T(D) \cap Y$ for $Y \in \mathcal{Y}$ the successors of $D$. We write $D \rightarrow C$, if $C$ is a successor of $D$. The successors are again subintervals of elements of $\mathcal{Y}$, so we can iterate the formation of successors. Set $\mathcal{D}_{1}=\mathcal{Y}$ and $\mathcal{D}_{k+1}=\mathcal{D}_{k} \cup\{C$ : there is $D \in \mathcal{D}_{k}$ with $D \rightarrow C$ \} for $k \geq 1$. Finally set $\mathcal{D}=\bigcup_{k=1}^{\infty} \mathcal{D}_{k}$.

In order to get a dynamical system, take disjoint copies $\hat{D}$ of the intervals $D \in \mathcal{D}$ and set $\hat{X}=\bigcup_{D \in \mathcal{D}} \hat{D}$. Let $q: \hat{D} \rightarrow[0,1]$ be the imbedding, such that $q: \hat{X} \rightarrow[0,1]$ is defined. If $D \in \mathcal{D}$ and $x \in D \cap \bigcup_{Y \in \mathcal{Y}} Y$ then $T(x)$ is defined and contained in $\bigcup_{D \rightarrow C} C$. For the corresponding point $\hat{x} \in \hat{D}$ we let $\hat{T}(\hat{x})$ be the point in $\bigcup_{D \rightarrow C} \hat{C}$ corresponding to $T(x)$. In this way we have defined $\hat{T}$ on the subset $q^{-1}\left(\bigcup_{Y \in \mathcal{Y}} Y\right)$ of $\hat{X}$, such that $q \circ \hat{T}=T \circ q$ holds. Note that $q^{-1}\left(\bigcup_{Y \in \mathcal{Y}} Y\right)$ contains $q^{-1}(R)$. If $H_{\mu}(\mathcal{Y})=-\sum_{Y \in \mathcal{Y}} \mu(Y) \log \mu(Y)<\infty$, it is shown in [8] (for finite $\mathcal{Y}$ in [11]) that there is a probability measure $\hat{\mu}$ on $\hat{X}$ with $\hat{\mu}\left(q^{-1}(R)\right)=1$, 
such that $\hat{T}$ is defined $\hat{\mu}$-almost everywhere and has the following properties

$$
\begin{aligned}
& \hat{\mu} \text { is a } \hat{T} \text {-invariant ergodic measure, } \\
& \hat{\mu}\left(q^{-1}(A)\right)=\mu(A) \text { for all Borel subsets } A \text { of }[0,1] .
\end{aligned}
$$

Set $\hat{\mathcal{Y}}=\{\hat{D}: D \in \mathcal{D}\}$. By the above construction, $\hat{\mathcal{Y}}$ is a finite or countable Markov partition of the dynamical system $(\hat{X}, \hat{T}, \hat{\mu})$. This explains the name Markov extension. For $n \geq 1$ let $\hat{Y}_{n}(\hat{x})$ be that element of $\hat{\mathcal{Y}}_{n}=\bigvee_{j=0}^{n-1} \hat{T}^{-j} \hat{\mathcal{Y}}$, which contains $\hat{x}$. If $\hat{x} \in q^{-1}(R)$ then $\hat{Y}_{n}(\hat{x})$ exists for all $n \geq 1$.

We investigate recurrence on the Markov extension.

Lemma 2.1. There is a set $\hat{M} \subset \hat{X}$ with $\hat{\mu}(\hat{M})=1$ such that $\limsup _{n \rightarrow \infty} \frac{1}{n} \tau\left(\hat{Y}_{n}(\hat{x})\right) \leq 1$ for all $\hat{x} \in \hat{M}$.

Proof. Choose $D \in \mathcal{D}$ with $\hat{\mu}(\hat{D})>0$. By the ergodic theorem there is a set $\hat{M}_{D} \subset \hat{X}$ with $\hat{\mu}\left(\hat{M}_{D}\right)=1$ such that $\lim _{n \rightarrow \infty} \frac{1}{n} \sum_{j=0}^{n-1} 1_{\hat{D}}\left(\hat{T}^{j}(\hat{x})\right)=\hat{\mu}(\hat{D})$ holds for all $\hat{x} \in \hat{M}_{D}$.

Let $\hat{x} \in \hat{D} \cap \hat{M}_{D}$ and let $n_{0}=0<n_{1}<n_{2}<\ldots$ be those integers $n$, for which $\hat{T}^{n}(\hat{x}) \in \hat{D}$ holds. The above result says that $\lim _{j \rightarrow \infty} \frac{j}{n_{j}}=\hat{\mu}(\hat{D})>0$. Since $\hat{\mathcal{Y}}$ is a Markov partition, we have either $\hat{D} \subset \hat{T}^{n_{j}}\left(\hat{Y}_{n_{j}}(\hat{x})\right)$ or $\hat{D} \cap \hat{T}^{n_{j}}\left(\hat{Y}_{n_{j}}(\hat{x})\right)=\emptyset$. Since $\hat{T}^{n_{j}}(\hat{x}) \in \hat{D} \cap \hat{T}^{n_{j}}\left(\hat{Y}_{n_{j}}(\hat{x})\right)$ we conclude that $\hat{D} \subset \hat{T}^{n_{j}}\left(\hat{Y}_{n_{j}}(\hat{x})\right)$ for $j \geq 1$. For arbitrary $n \geq 1$ choose $j$ such that $n_{j-1}<n \leq n_{j}$. Then $\hat{Y}_{n_{j}}(\hat{x}) \subset \hat{Y}_{n}(\hat{x})$ and $\hat{x} \in \hat{D} \subset \hat{T}^{n_{j}}\left(\hat{Y}_{n}(\hat{x})\right)$ follows. Since $\hat{x} \in \hat{Y}_{n}(\hat{x})$ we get $\tau\left(\hat{Y}_{n}(\hat{x})\right) \leq n_{j}$ and $\frac{1}{n} \tau\left(\hat{Y}_{n}(\hat{x})\right) \leq \frac{n_{j}}{n_{j-1}}$. Furthermore, $\lim _{j \rightarrow \infty} \frac{n_{j}}{n_{j-1}}=\lim _{j \rightarrow \infty} \frac{j}{j-1} \frac{n_{j}}{j} \frac{j-1}{n_{j-1}}=1$. This implies $\limsup _{n \rightarrow \infty} \frac{1}{n} \tau\left(\hat{Y}_{n}(\hat{x})\right) \leq 1$.

Set $\mathcal{D}^{*}=\{D \in \mathcal{D}: \hat{\mu}(\hat{D})>0\}$ and $\hat{M}=\bigcup_{D \in \mathcal{D}^{*}}\left(\hat{D} \cap \hat{M}_{D}\right)$. We have shown, that $\limsup _{n \rightarrow \infty} \frac{1}{n} \tau\left(\hat{Y}_{n}(\hat{x})\right) \leq 1$ holds for all $\hat{x} \in \hat{M}$. Since $\hat{\mu}\left(\hat{D} \cap \hat{M}_{D}\right)=\hat{\mu}(\hat{D})$ we get

$$
\hat{\mu}(\hat{M})=\sum_{D \in \mathcal{D}^{*}} \hat{\mu}\left(\hat{D} \cap \hat{M}_{D}\right)=\sum_{D \in \mathcal{D}^{*}} \hat{\mu}(\hat{D})=\sum_{D \in \mathcal{D}} \hat{\mu}(\hat{D})=\hat{\mu}(\hat{X})=1
$$

and the proof is finished.

We bring down this result to the original dynamical system.

Lemma 2.2. We have $\lim \sup _{n \rightarrow \infty} \frac{1}{n} \tau\left(Y_{n}(x)\right) \leq 1$ for $\mu$-almost all $x \in[0,1]$.

Proof. Let $\hat{M}$ be as in Lemma 2.1 and set $M=q(\hat{M}) \cap R$. Then $q^{-1}(M) \supset$ $\hat{M} \cap q^{-1}(R)$ and hence $\mu(M)=\hat{\mu}\left(q^{-1}(M)\right)=1$.

For $x \in M$ there is $\hat{x} \in \hat{M}$ with $q(\hat{x})=x$. Suppose that $D_{n} \in \mathcal{D}$ is such that $\hat{T}^{n}(\hat{x}) \in \hat{D}_{n}$ for all $n \geq 0$. We have $q\left(\hat{D}_{n}\right)=D_{n}$. By the definition of successor and 
because of $q \circ \hat{T}=T \circ q$ there are $U_{0}, U_{1}, \cdots \in \mathcal{Y}$, such that $T^{n}(x) \in D_{n} \subset U_{n}$ for $n \geq 0$. It follows that $Y_{n}(x)=\bigcap_{j=0}^{n-1} T^{-j} U_{j}$ and $\hat{Y}_{n}(\hat{x})=\bigcap_{j=0}^{n-1} \hat{T}^{-j} \hat{D}_{j}$. We get $q\left(\hat{Y}_{n}(\hat{x})\right)=\bigcap_{j=0}^{n-1} T^{-j} D_{j} \subset Y_{n}(x)$. If $\tau\left(\hat{Y}_{n}(\hat{x})\right)=k$, then $\hat{T}^{k}\left(\hat{Y}_{n}(\hat{x})\right) \cap \hat{Y}_{n}(\hat{x}) \neq \emptyset$, and since $q \circ \hat{T}^{k}=T^{k} \circ q$ holds we get $T^{k}\left(Y_{n}(x)\right) \cap Y_{n}(x) \neq \emptyset$. Hence $\tau\left(Y_{n}(x)\right) \leq$ $\tau\left(\hat{Y}_{n}(\hat{x})\right)$. It follows from Lemma 2.1 that $\lim _{\sup } \rightarrow \infty \frac{1}{n} \tau\left(Y_{n}(x)\right) \leq 1$ holds for all $x \in M$, which means for $\mu$-almost all $x$.

Lemma 2.3. Let $T$ be a piecewise monotonic transformation, which has only finitely many or no attracting periodic orbits. Suppose that $\varphi=-\log \left|T^{\prime}\right|$ is either in $C$ or in $D^{p}$ for some $p \geq 1$. Let $\mu$ be an ergodic $T$-invariant measure with $h_{\mu}>0$. Then $\mu(\varphi) \leq 0$ and $\varphi \in L^{1}(\mu)$.

Proof. Suppose that $\mu(\varphi)>0$ holds. There is a continuous function $\psi:[0,1] \rightarrow$ $\mathbb{R}$ satisfying $\psi \leq \varphi$ and $\mu(\psi)>0$. Let $(\hat{X}, \hat{T}, \hat{\mu})$ be the Markov extension of $([0,1], T, \mu)$ with respect to the partition $\mathcal{Z}$ and let $q$ be the corresponding projection. By the ergodic theorem, there is $\hat{x} \in \hat{X}$, such that $\frac{1}{n} \sum_{j=0}^{n-1} \delta_{\hat{T}^{j}(\hat{x})}$ converges weakly to $\hat{\mu}$. Since $\hat{\mu}$ is ergodic and has nonzero entropy, the set of all $\hat{y}$, for which $\bigcap_{j=1}^{\infty} \hat{Y}_{n}(\hat{y})$ is a nondegenerate interval, has $\hat{\mu}$-measure zero, and hence we can assume that $\bigcap_{j=1}^{\infty} \hat{Y}_{n}(\hat{x})=\{\hat{x}\}$. There is a sequence $D_{0} D_{1} D_{2} \ldots$ in $\mathcal{D}$ such that $\hat{T}^{k}(\hat{x}) \in \hat{D}_{k}$ for $k \geq 0$. Since $\hat{\mathcal{Y}}$ is a Markov partition, we have $\hat{T}\left(\hat{D}_{k}\right) \supset \hat{D}_{k+1}$ for $k \geq 0$. There is $D \in \mathcal{D}$ with $\hat{\mu}(\hat{D})>0$, and hence $D$ occurs infinitely often in this sequence. Choose $n_{1}<n_{2}<n_{3}<\ldots$ such that $D_{n_{k}}=D$. For every $m$ there is a point $\hat{y}$ of period $n_{m}-n_{1}$ with $\hat{T}^{j}(\hat{y}) \in \hat{D}_{n_{1}+j}$ for $0 \leq j \leq n_{m}-n_{1}$. Let $\hat{v}_{m}$ be the periodic-orbit-measure supported by the periodic orbit of $\hat{y}$. Then the sequence $\hat{v}_{m}$ converges weakly to $\hat{\mu}$. Since $q: \hat{X} \rightarrow[0,1]$ is continuous, the measures $v_{m}=\hat{v}_{m} \circ q^{-1}$ are periodic-orbit-measures on $([0,1], T)$, which converge weakly to $\mu$. Since $\psi$ is continuous, there is $m_{0}$ with $v_{m}(\psi)>0$ for $m \geq m_{0}$. Because of $\varphi \geq \psi$ we have also $v_{m}(\varphi)>0$ for $m \geq m_{0}$. This implies, that the periodic orbits supporting the measures $v_{m}$ for $m \geq m_{0}$ are attracting, contradicting the assumption, that at most finitely many attracting periodic orbits exist. This contradiction shows that $\mu(\varphi) \leq 0$. Since we assume that $\varphi$ is in $C$ or in $D^{p}$, it follows that $\varphi$ is bounded below and hence in $L^{1}(\mu)$.

Theorem 2.4. Let $T$ be a piecewise monotonic transformation, which has only finitely many or no attracting periodic orbits. Suppose that $\varphi=-\log \left|T^{\prime}\right|$ is either in $C$ or in $D^{p}$ for some $p \geq 1$. Let $\mu$ be an ergodic $T$-invariant measure with $h_{\mu}>0$. Then $\chi_{\mu}:=-\mu(\varphi)$ is greater than zero and $\lim _{r \rightarrow 0} \frac{\tau\left(B_{r}(x)\right)}{-\log r}=\frac{1}{\chi_{\mu}}$ holds for $\mu$-almost all $x$.

Proof. By Lemma 2.3 we have $\varphi \in L^{1}(\mu)$ and by Theorem 1 in [6] we get $\chi_{\mu}=$ $-\mu(\varphi)>0$. Choose $\varepsilon \in\left(0, \frac{\chi_{\mu}}{2}\right)$. By Lemma 1 in [8] there is a finite or countable 
collection $\mathcal{Y}$ of pairwise disjoint intervals, such that

$$
\begin{aligned}
& \mu\left(\bigcup_{Y \in \mathcal{Y}} Y\right)=1 \\
& \sup _{x \in Y} \varphi(x)-\inf _{x \in Y} \varphi(x)<\varepsilon \text { for every } Y \in \mathcal{Y} ; \\
& H_{\mu}(\mathcal{Y})=-\sum_{Y \in \mathcal{Y}} \mu(Y) \log \mu(Y)<\infty .
\end{aligned}
$$

Now let $M_{\varepsilon}$ be a set of $\mu$-measure one, such that $\lim _{\sup } \rightarrow \infty \frac{1}{n} \tau\left(Y_{n}(x)\right) \leq 1$ and that $\lim _{n \rightarrow \infty} \frac{1}{n} \sum_{j=0}^{n-1} \varphi\left(T^{j} x\right)=\mu(\varphi)=-\chi_{\mu}$ for all $x \in M_{\varepsilon}$. This is possible because of Lemma 2.2 and the ergodic theorem. For $x \in M_{\varepsilon}$ we get by the mean value theorem and as $T^{n}\left(Y_{n}(x)\right) \subset[0,1]$ that

$$
\log \left|Y_{n}(x)\right| \leq \sup _{y \in Y_{n}(x)} \sum_{j=0}^{n-1} \varphi\left(T^{j} y\right)+\log \left|T^{n}\left(Y_{n}(x)\right)\right| \leq \sup _{y \in Y_{n}(x)} \sum_{j=0}^{n-1} \varphi\left(T^{j} y\right) .
$$

Since $\sup _{U} \varphi-\inf _{U} \varphi<\varepsilon$ for every $U \in \mathcal{Y}$ we get $\sum_{j=0}^{n-1} \varphi\left(T^{j} y\right) \leq \sum_{j=0}^{n-1} \varphi\left(T^{j} x\right)+$ $n \varepsilon$, if $y \in Y_{n}(x)$, and hence $\log \left|Y_{n}(x)\right| \leq \sum_{j=0}^{n-1} \varphi\left(T^{j} x\right)+n \varepsilon$. It follows that

$$
\lim _{n \rightarrow \infty} \frac{1}{n} \log \left|Y_{n}(x)\right| \leq-\chi_{\mu}+\varepsilon .
$$

In particular we have $\lim _{n \rightarrow \infty}\left|Y_{n}(x)\right|=0$, as $-\chi_{\mu}+\varepsilon<0$.

For $x \in M_{\varepsilon}$ and $r>0$ let $n$ be such that $\left|Y_{n}(x)\right| \leq r<\left|Y_{n-1}(x)\right|$. This implies $Y_{n}(x) \subseteq B_{r}(x)$, and therefore

$$
\frac{\tau\left(B_{r}(x)\right)}{-\log r} \leq \frac{\tau\left(Y_{n}(x)\right)}{-\log \left|Y_{n-1}(x)\right|}=\frac{\frac{1}{n} \tau\left(Y_{n}(x)\right)}{-\frac{1}{n} \log \left|Y_{n-1}(x)\right|} .
$$

There is $n_{0}$, such that $\frac{1}{n} \tau\left(Y_{n}(x)\right)<1+\varepsilon$ and $-\frac{1}{n} \log \left|Y_{n-1}(x)\right|>\chi_{\mu}-2 \varepsilon$ for $n \geq n_{0}$. For $r<\left|Y_{n_{0}}(x)\right|$ we get $\frac{\tau\left(B_{r}(x)\right)}{-\log r}<\frac{1+\varepsilon}{\chi_{\mu}-2 \varepsilon}$ and hence also

$$
\limsup _{r \rightarrow 0} \frac{\tau\left(B_{r}(x)\right)}{-\log r} \leq \frac{1+\varepsilon}{\chi_{\mu}-2 \varepsilon} .
$$

Now set $M=\bigcap_{k=1}^{\infty} M_{1 / k}$. Then we have $\mu(M)=1$ and $\lim \sup _{r \rightarrow 0} \frac{\tau\left(B_{r}(x)\right)}{-\log r} \leq \frac{1}{\chi_{\mu}}$ for all $x \in M$, which means for $\mu$-almost all $x$.

Theorem 2 in [15] says that $\liminf _{r \rightarrow 0} \frac{\tau\left(B_{r}(x)\right)}{-\log r} \geq \frac{1}{\chi_{\mu}}$ holds for $\mu$-almost all $x$. It is proved there first for cylinder sets with respect to a partition for general dynamical systems and then an approximation procedure as above is used. 
In the proof of the result on the dimension of a measure we shall use centered covers. For $\varepsilon>0$ we call $\mathcal{F}$ a centered $\delta$-cover of $A$, if $A \subseteq \bigcup_{C \in \mathcal{F}} C$ and for every $C \in \mathcal{F}$ there exists an $x \in A$ and an $\alpha \in(0, \delta]$ with $C=(x-\alpha, x+\alpha)$. The next lemma shows that we can use centered covers instead of covers.

Lemma 2.5. For $A \subset[0,1]$ let $\tilde{v}_{s, t}(A)$ be defined in the same way as $v_{s, t}(A)$ but with centered covers instead of covers. Then $v_{s, t}(A) \leq \tilde{v}_{s, t}(A) \leq 2 v_{s, t}(A)$ for $s, t \geq 0$.

Proof. Since a centered $\delta$-cover is also a $\delta$-cover, it follows that $v_{s, t}(A) \leq \tilde{v}_{s, t}(A)$.

Let $I$ be an interval of length $\leq \delta$ with $I \cap A \neq \emptyset$. Let $I_{1}$ be the left half and $I_{2}$ the right half of $I$. If $I_{1} \cap A \neq \emptyset$, there is $x_{1} \in I_{1} \cap A$ with $I_{1} \subset B_{r_{1}}\left(x_{1}\right) \subset I$, where $r_{1}$ is the distance from $x_{1}$ to the left endpoint of $I$. If $I_{1} \cap A=\varnothing$, set $B_{r_{1}}\left(x_{1}\right)=\emptyset$. Similarly, if $I_{2} \cap A \neq \emptyset$, there is $x_{2} \in I_{2} \cap A$ with $I_{2} \subset B_{r_{2}}\left(x_{2}\right) \subset I$, where $r_{2}$ is the distance from $x_{2}$ to the right endpoint of $I$. If $I_{2} \cap A=\varnothing$, set $B_{r_{2}}\left(x_{2}\right)=\emptyset$. We have $A \cap\left(B_{r_{1}}\left(x_{1}\right) \cup B_{r_{2}}\left(x_{2}\right)\right)=A \cap I$. If $\mathcal{C}$ is a $\delta$-cover of $A$, for each $I \in \mathcal{C}$ choose $B_{r_{1}}\left(x_{1}\right)$ and $B_{r_{2}}\left(x_{2}\right)$ as above. The nonempty sets among them form a centered $\delta$-cover $\tilde{\mathcal{C}}$ of $A$ such that $\sum_{\tilde{C} \in \tilde{\mathcal{C}}} e^{-s \tau(\tilde{C})}|\tilde{C}|^{t} \leq 2 \sum_{C \in \mathcal{C}} e^{-s \tau(C)}|C|^{t}$, since $U \subset V$ implies $\tau(U) \geq \tau(V)$ and $|U| \leq|V|$. From this inequality we get $\tilde{v}_{s, t}(A) \leq 2 v_{s, t}(A)$.

Theorem 2.6. Let $T$ be a piecewise monotonic transformation, which has only finitely many or no attracting periodic orbits. Suppose that $\varphi=-\log \left|T^{\prime}\right|$ is either in $C$ or in $D^{p}$ for some $p \geq 1$. Let $\mu$ be an ergodic $T$-invariant measure with $h_{\mu}>0$. Then for every $s \in\left[0, h_{\mu}\right)$ we have $d_{s}(\mu)=\frac{h_{\mu}-s}{\chi_{\mu}}$, where $\chi_{\mu}=-\mu(\varphi)$.

Proof. Set $\alpha=\frac{h_{\mu}}{\chi_{\mu}}$ and $\beta=\frac{1}{\chi_{\mu}}$. By Theorem 2.4 we have $\chi_{\mu}>0$ and $\lim _{r \rightarrow 0} \frac{\tau\left(B_{r}(x)\right)}{-\log r}=$ $\beta$ for $\mu$-almost all $x$. By Theorem 1 in [7] we have $\lim _{r \rightarrow 0} \frac{\log \mu\left(B_{r}(x)\right)}{\log r}=\alpha$ for $\mu$ almost all $x$.

In order to prove $d_{s}(\mu) \leq \alpha-s \beta$ choose an arbitrary $t>\alpha-s \beta$ and set $\varepsilon=\frac{1}{2}(t-\alpha+s \beta)$. Since $s<h_{\mu}$, we have $t>0$. For $k \in \mathbb{N}$ define

$M_{k}=\left\{x \in[0,1]: \mu\left(B_{r}(x)\right)^{\frac{t-\varepsilon}{\alpha}} \geq \frac{1}{k} r^{t}\right.$ and $\mu\left(B_{r}(x)\right)^{\frac{s \beta-\varepsilon}{\alpha}} \geq \frac{1}{k} e^{-s \tau\left(B_{r}(x)\right)}$ for all $\left.r \in(0,1)\right\}$

For $\mu$-almost all $x \in[0,1]$ there exists an $r_{0}(x)>0$ such that

$$
\begin{gathered}
\frac{t-\varepsilon}{\alpha} \frac{\log \mu\left(B_{r}(x)\right)}{\log r} \leq t \quad \text { and } \\
\frac{s \beta-\varepsilon}{\alpha} \frac{-\log \mu\left(B_{r}(x)\right)}{\tau\left(B_{r}(x)\right)}=\frac{s \beta-\varepsilon}{\alpha} \frac{\log \mu\left(B_{r}(x)\right)}{\log r} \frac{-\log r}{\tau\left(B_{r}(x)\right)} \leq s
\end{gathered}
$$

for all $r \in\left(0, r_{0}(x)\right)$. These are the inequalities in the definition of $M_{k}$ with $k=1$. Hence for $\mu$-almost all $x \in[0,1]$ there is a $k \in \mathbb{N}$ with $x \in M_{k}$, and therefore $\mu\left(\bigcup_{k=1}^{\infty} M_{k}\right)=1$. 
Fix $k \in \mathbb{N}$ and $\delta \in(0,1)$, and let $\mathcal{C}$ be a centered $\delta$-cover of $M_{k}$. Since each $C \in \mathcal{C}$ is a ball with center in $M_{k}$ we get by the definition of $M_{k}$

$$
\sum_{C \in \mathcal{C}} e^{-s \tau(C)}|C|^{t} \leq 2^{t} k^{2} \sum_{C \in \mathcal{C}} \mu(C)^{\frac{s \beta-\varepsilon}{\alpha}} \mu(C)^{\frac{t-\varepsilon}{\alpha}}=2^{t} k^{2} \sum_{C \in \mathcal{C}} \mu(C) \leq 2^{t+1} k^{2} .
$$

The last inequality follows, since we can assume that each $x \in[0,1]$ is contained in at most two element of $\mathcal{C}$. Otherwise we can cancel elements from $\mathcal{C}$ and have still a cover. This implies that $\tilde{v}_{s, t}\left(M_{k}\right)<2^{t+1} k^{2}$. By Lemma 2.5 we get also $v_{s, t}\left(M_{k}\right)<2^{t+1} k^{2}$. By the definition of dimension we have $d_{s}\left(M_{k}\right) \leq t$. For $u>t$ we have therefore $v_{s, u}\left(M_{k}\right)=0$ for all $k$, and as $v_{s, u}$ is an outer measure, we get $v_{s, u}\left(\bigcup_{k=1}^{\infty} M_{k}\right)=0$. It follows that $d_{s}\left(\bigcup_{k=1}^{\infty} M_{k}\right) \leq t$ and hence $d_{s}(\mu) \leq t$, since $\mu\left(\bigcup_{k=1}^{\infty} M_{k}\right)=1$. As $t$ can be chosen arbitrarily close to $\alpha-s \beta$, we get $d_{S}(\mu) \leq \alpha-s \beta$.

In order to prove that $\alpha-s \beta \leq d_{s}(\mu)$, it suffices to show $\mu(L)=0$ for every Borel set $L \subseteq[0,1]$ with $d_{s}(L)<\alpha-s \beta$. Let $L$ be such a set. Since $s<h_{\mu}$, we have $\alpha-s \beta>0$. Choose $t>0$ with $d_{s}(L)<t<\alpha-s \beta$ and set $\varepsilon=\frac{1}{2}(\alpha-s \beta-t)$. For $k \in \mathbb{N}$ define

$$
L_{k}=\left\{x \in L: \mu\left(B_{r}(x)\right)^{\frac{t+\varepsilon}{\alpha}} \leq k r^{t} \text { and } \mu\left(B_{r}(x)\right)^{\frac{s \beta+\varepsilon}{\alpha}} \leq k e^{-s \tau\left(B_{r}(x)\right)} \text { for all } r \in(0,1)\right\}
$$

For $\mu$-almost all $x \in L$ there is an $r_{0}(x)>0$ such that

$$
\begin{gathered}
\frac{t+\varepsilon}{\alpha} \frac{\log \mu\left(B_{r}(x)\right)}{\log r} \geq t \text { and } \\
\frac{s \beta+\varepsilon}{\alpha} \frac{-\log \mu\left(B_{r}(x)\right)}{\tau\left(B_{r}(x)\right)}=\frac{s \beta+\varepsilon}{\alpha} \frac{\log \mu\left(B_{r}(x)\right)}{\log r} \frac{-\log r}{\tau\left(B_{r}(x)\right)} \geq s
\end{gathered}
$$

holds for all $r \in\left(0, r_{0}(x)\right)$. These are the inequalities in the definition of $L_{k}$ with $k=1$. Hence for $\mu$-almost all $x \in L$ there is a $k \in \mathbb{N}$ with $x \in L_{k}$, and therefore $\mu(L)=\mu\left(\bigcup_{k=1}^{\infty} L_{k}\right)$. It remains to show $\mu\left(L_{k}\right)=0$ for all $k \in \mathbb{N}$.

To this end fix $k \in \mathbb{N}$ and $\eta>0$. Since $d_{s}\left(L_{k}\right) \leq d_{S}(L)<t$ we have $v_{s, t}\left(L_{k}\right)=0$ and hence also $\tilde{v}_{s, t}\left(L_{k}\right)=0$ by Lemma 2.5 . There exists a $\delta \in(0,1)$ and a centered $\delta$-cover $\mathcal{C}$ of $L_{k}$ with

$$
\sum_{C \in \mathcal{C}} e^{-s \tau(C)}|C|^{t}<\frac{2^{t}}{k^{2}} \eta
$$

Since each $C \in \mathcal{C}$ is a ball with center in $L_{k}$ we get by the definition of $L_{k}$ that

$$
\mu\left(L_{k}\right) \leq \sum_{C \in \mathcal{C}} \mu(C)=\sum_{C \in \mathcal{C}} \mu(C)^{\frac{s \beta+\varepsilon}{\alpha}} \mu(C)^{\frac{t+\varepsilon}{\alpha}} \leq \frac{k^{2}}{2^{t}} \sum_{C \in \mathcal{C}} e^{-s \tau(C)}|C|^{t}<\eta .
$$

As $\eta>0$ was arbitrary we get $\mu\left(L_{k}\right)=0$, which completes the proof.

Remark 2.7. The statement in Theorem 2.6 holds also for $s<0$. This can be shown by the method developed in chapter 5 of [16], which is based on a result in [4]. But it seems that the proof for $s>0$ cannot be given by this method. One gets only an inequality in this case. 


\section{Dimension of an invariant set}

In order to investigate the dimension of a closed invariant set $A$ in the dynamical system $([0,1], T)$, we assume that $T$ is expanding, this means that inf $\left|T^{\prime}\right|>1$. Furthermore, we assume that $T^{\prime} \in C$. This implies that $\varphi=-\log \left|T^{\prime}\right|$ is also in $C$. For a function $f \in C$ we define

$$
p(T \mid A, f)=\limsup _{n \rightarrow \infty} \frac{1}{n} \log \sum_{Z \in \mathcal{Z}_{n}(A)} \sup _{x \in Z \cap A} e^{S_{n} f(x)}
$$

where $S_{n} f(x)=\sum_{j=0}^{n-1} f\left(T^{j} x\right)$ and $\mathcal{Z}_{n}(A)=\left\{Z \in \mathcal{Z}_{n}: Z \cap A \neq \emptyset\right\}$. If we consider the dynamical system $([0,1], T)$ as a shift space, this is the usual definition of pressure. In particular, the pressure does not change, if we refine $\mathcal{Z}$, and the variational principle holds. These and other properties of the pressure can be found in [17]. We define the function $\pi:[0, \infty) \rightarrow \mathbb{R}$ by

$$
\pi(t)=p(T \mid A, t \varphi) .
$$

For $t_{1}<t_{2}$ we have $t_{2} \varphi \leq t_{1} \varphi+\left(t_{2}-t_{1}\right) \sup \varphi$ and hence $\pi\left(t_{2}\right) \leq \pi\left(t_{1}\right)+$ $\left(t_{2}-t_{1}\right) \sup \varphi$. Since $\sup \varphi<0$ this implies that $\pi$ is strictly decreasing and that $\lim _{t \rightarrow \infty} \pi(t)=-\infty$. Furthermore, $\pi(0)=h_{\text {top }}(T \mid A) \geq 0$. Hence for $s \in\left[0, h_{\text {top }}(T \mid A)\right]$ there is a unique $\tilde{t} \geq 0$ with $\pi(\tilde{t})=s$, with $\pi(t)>s$ for $t<\tilde{t}$ and with $\pi(t)<s$ for $t>\tilde{t}$. We denote this $\tilde{t}$ by $z_{s}(A)$. If $s<h_{\text {top }}(T \mid A)$ then $z_{s}(A)>0$.

Theorem 3.1. Let $T$ be an expanding piecewise monotonic transformation on $[0,1]$, such that $T^{\prime} \in C$, and $A$ a closed invariant subset with $h_{\text {top }}(T \mid A)>0$. For $s \in\left[0, h_{\text {top }}(T \mid A)\right)$ we have then $d_{s}(A)=z_{s}(A)$.

Proof. Since $s<h_{\text {top }}(T \mid A)$ we have $z_{s}(A)>0$. Choose $t \in\left(0, z_{s}(A)\right)$. Since $t<z_{s}(A)$, we get $p(T \mid A, t \varphi)=\pi(t)>s$. By the variational principle there is an ergodic invariant measure $\mu$ on $A$ satisfying $h_{\mu}+t \mu(\varphi)>s$. Because of $\mu(\varphi) \leq \sup \varphi<0$ we get $h_{\mu}>s$. Since $\varphi \in C$ we can apply Theorem 2.6 and get $d_{s}(\mu)=\frac{h_{\mu}-s}{\chi_{\mu}}$. Since $h_{\mu}-s>-t \mu(\varphi)=t \chi_{\mu}$ we get $d_{s}(\mu)>t$. This implies $d_{s}(A)>t$. As $t$ can be chosen arbitrarily close to $z_{s}(A)$, the inequality $d_{s}(A) \geq z_{s}(A)$ is shown.

Choose $t>z_{S}(A)$. Then $t>0$ and $\pi(t)<s$. Fix $\varepsilon \in\left(0, \frac{s-\pi(t)}{t}\right)$. We choose points $0=d_{0}<d_{1}<\cdots<d_{K}=1$ containing $c_{0}, c_{1}, \ldots, c_{N}$ and set $\mathcal{Z}=\left\{\left(d_{0}, d_{1}\right), \ldots,\left(d_{K-1}, d_{K}\right)\right\}$, such that $T \mid Z$ is monotone and $\sup _{x \in Z} \varphi(x)-$ $\inf _{x \in Z} \varphi(x)<\varepsilon$ for every $Z \in \mathcal{Z}$. If we define the pressure with respect to this finer partition $\mathcal{Z}$ we get the same as for the original $\mathcal{Z}$. Set $\alpha=\frac{s-\pi(t)-\varepsilon t}{2}>0$ and $p_{n}=e^{n(t \varepsilon-s)} \sum_{Z \in \mathcal{Z}_{n}(A)} \sup _{Z \cap A} e^{t S_{n} \varphi}$ for $n \geq 1$. Then

$\limsup _{n \rightarrow \infty} \frac{1}{n} \log p_{n}=t \varepsilon-s+\limsup _{n \rightarrow \infty} \frac{1}{n} \log \sum_{Z \in \mathcal{Z}_{n}(A)} \sup _{Z \cap A} e^{t S_{n} \varphi}=t \varepsilon-s+\pi(t)=-2 \alpha$.

Hence there is a constant $c>0$, such that $p_{n} \leq c e^{-n \alpha}$ holds for all $n \geq 1$. 
Set $\mathcal{Z}_{n}^{k}(A)=\left\{Z \in \mathcal{Z}_{n}(A): \tau(Z)=k\right\}$ and $\mathcal{Z}_{n}^{n}(A)=\left\{Z \in \mathcal{Z}_{n}(A): \tau(Z) \geq\right.$ $n$ \}. Suppose that $Z=\bigcap_{j=0}^{n-1} T^{-j} Z_{j} \in \mathcal{Z}_{n}^{k}(A)$, where $Z_{0}, Z_{1}, \ldots, Z_{n-1} \in \mathcal{Z}$ and $k<n$. Then $T^{k}(Z) \cap Z \neq \varnothing$ and hence $Z_{j+k}=Z_{j}$ for $0 \leq j \leq n-k-1$. Set $\tilde{Z}=\bigcap_{j=0}^{k-1} T^{-j} Z_{j}$. Then $Z \subset \tilde{Z}$ and $\tilde{Z} \in \mathcal{Z}_{k}(A)$, as $A \cap Z \neq \emptyset$ and hence $A \cap \tilde{Z} \neq \varnothing$. Furthermore, the map $Z \mapsto \tilde{Z}$ from $\mathcal{Z}_{n}^{k}(A)$ to $\mathcal{Z}_{k}(A)$ is injective, since $Z_{k}, \ldots, Z_{n-1}$ are determined by $Z_{1}, \ldots, Z_{k-1}$. For $k=n$ we set $\tilde{Z}=Z$ and these results are trivial.

For each $Z \in \mathcal{Z}_{k}(A)$ we get by the mean value theorem that

$$
|Z| \leq\left|T^{k} Z\right| \sup _{x \in Z}\left|T^{(k)}(x)\right|-1=\left|T^{k} Z\right| \sup _{x \in Z} e^{S_{k} \varphi(x)} .
$$

Since $T^{k} Z \subset[0,1]$ we have $\left|T^{k} Z\right| \leq 1$. Since $\sup _{U} \varphi-\inf _{U} \varphi<\varepsilon$ for every $U \in \mathcal{Z}$ we have $S_{k} \varphi(x)-S_{k} \varphi(y) \leq k \varepsilon$ whenever $x$ and $y$ are in $Z$. As $A \cap Z \neq \emptyset$ it follows that

$$
|Z| \leq \sup _{x \in A \cap Z} e^{S_{k} \varphi(x)+k \varepsilon} .
$$

If $Z \in \mathcal{Z}_{n}^{k}(A)$ and $\tilde{Z}$ as above, we have $|Z| \leq|\tilde{Z}|$ since $Z \subset \tilde{Z}$. Hence we get

$$
\begin{aligned}
\sum_{Z \in \mathcal{Z}_{n}(A)} e^{-s \tau(Z)}|Z|^{t} & \leq \sum_{k=1}^{n} e^{-s k} \sum_{Z \in \mathcal{Z}_{n}^{k}(A)}|Z|^{t} \leq \sum_{k=1}^{n} e^{-s k} \sum_{\tilde{Z} \in \mathcal{Z}_{k}(A)}|\tilde{Z}|^{t} \\
& \leq \sum_{k=1}^{n} e^{-s k} \sum_{\tilde{Z} \in \mathcal{Z}_{k}(A)} \sup _{A \cap \tilde{Z}} e^{t S_{k} \varphi+t k \varepsilon} \\
& =\sum_{k=1}^{n} p_{k} \leq \sum_{k=1}^{\infty} p_{k} \leq c \sum_{k=1}^{\infty} e^{-k \alpha} \leq \frac{c e^{-\alpha}}{1-e^{-\alpha}}
\end{aligned}
$$

Now $\mathcal{Z}_{n}(A)$ covers the set $A$ except for possibly finitely many points. We can cover these finitely many points by intervals $I_{1}, I_{2}, \ldots, I_{j}$ of arbitrary small length, such that $\sum_{i=1}^{j} e^{-s \tau\left(I_{i}\right)}\left|I_{i}\right|^{t}<1$ holds. Since $T$ is expanding, for every $\delta>0$ there is $n$ such that the diameter of all intervals in $\mathcal{Z}_{n}(A)$ is less than $\delta$. Hence for every $\delta>0$ we have found a $\delta$-cover $\mathcal{C}$ of $A$ with $\sum_{C \in \mathcal{C}} e^{-s \tau(C)}|C|^{t}<\frac{c e^{-\alpha}}{1-e^{-\alpha}}+1$. This implies that $v_{s, t}(A) \leq \frac{c e^{-\alpha}}{1-e^{-\alpha}}+1$ and $d_{s}(A) \leq t$ follows. We can choose $t$ arbitrarily close to $z_{s}(A)$ so that $\bar{d}_{s}(A) \leq z_{s}(A)$ is shown.

\section{References}

[1] V. Afraimovich, Pesins dimension for Poincaré recurrences, Chaos 7 (1997), 12-20.

[2] V. Afraimovich and J. URIAS, Dimension-like characteristics of invariant sets in dynamical systems, In: "Dynamics and randomness", A. Maass, S. Martinez and J. San Martin (eds.), Kluwer Academic Publishers, Dordrecht, 2002, pp. 1-30. 
[3] V. Afraimovich, J.-R. Chazottes and B. Saussol, Local dimensions for Poincaré recurrence, Electron. Res. Announc. Amer. Math. Soc. 6 (2000), 64-74.

[4] J.-R. Chazottes and B. Saussol, On pointwise dimensions and spectra of measures, C. R. Acad. Sci. Paris Ser. I Math. 333 (2001), 719-723.

[5] F. Hofbauer, Piecewise invertible dynamical systems, Probab. Theory Related Fields $\mathbf{7 2}$ (1986), 359-386.

[6] F. HofBAUER, An inequality for the Ljapunov exponent of an ergodic invariant measure for a piecewise monotonic map on the interval, In: "Lyapunov exponents", Proceedings, Oberwolfach, 1990, Lecture Notes in Mathematics 1486, L. Arnold, H. Crauel and J.-P. Eckmann (eds.), Springer, Berlin, 1991, pp. 227-231.

[7] F. HofBAUER, Local dimension for piecewise monotonic maps on the interval, Ergod. Theory Dynam. Systems 15 (1995), 1119-1142.

[8] F. HoFBAUER and P. RAITH, The Hausdorff dimension of an ergodic invariant measure for a piecewise monotonic map of the interval, Canad. J. Math. 35 (1992), 84-98.

[9] F. Hofbauer, P. RAith and T. STEInberger, Multifractal dimensions for invariant subsets of piecewise monotonic interval maps, Fund. Math. 176 (2003), 209-232.

[10] G. KELlER, Markov extensions, zeta functions, and Fredholm theory for piecewise invertible dynamical systems, Trans. Amer. Math. Soc. 314 (1989), 433-497.

[11] G. Keller, Lifting measures to Markov extensions, Monatsh. Math. 108 (1989), 183-200.

[12] W. DE MELO and S. VAN STRIEN, "One-dimensional dynamics", Springer-Verlag BerlinHeidelberg-New York, 1993.

[13] L. Olsen, A multifractal formalism, Adv. Math. 116 (1995), 82-196.

[14] Y. PESIN, "Dimension theory in dynamical systems: Contemporary views and applications", The University of Chicago Press Chicago and London, 1997.

[15] B. Saussol, S. Troubetzkoy and S. Vaienti, Recurrence, dimensions and Lyapunov exponents, J. Statist. Phys. 106 (2002), 623-634.

[16] B. Saussol, S. Troubetzkoy and S. Vaienti, Recurrence and Lyapunov exponents, Moscow Math. J. 3 (2003), 189-203.

[17] P. WALters, "An introduction to ergodic theory", Springer-Verlag Berlin-Heidelberg-New York, 1982.

Fakultät für Mathematik

Universität Wien

Nordbergstraße 15

A 1090 Wien, Austria

franz.hofbauer@univie.ac.at 